\title{
Erratum to: Geometric models for the spectra of certain Gelfand pairs associated with Heisenberg groups
}

\author{
Chal Benson • Gail Ratcliff
}

Published online: 27 December 2012

(C) Fondazione Annali di Matematica Pura ed Applicata and Springer-Verlag Berlin Heidelberg 2012

\section{Erratum to: Annali di Matematica \\ DOI 10.1007/s10231-011-0234-x}

The paper contains an error which necessitates some revisions to the proof of our main result, Theorem 1.2. In fact, the polynomial functions $\widehat{D}_{\alpha} \in \mathbb{C}\left[\mathfrak{a}^{*}\right](\alpha \in \Lambda)$, discussed in Section 2.2 , need not, in general, be invariant under the little Weyl group $W_{\circ}$ as stated. One needs to introduce a " $\varrho$-shift" (half the sum of the positive roots) to achieve $W_{\circ}$-invariance. This means that although $\operatorname{top}\left(\widehat{D}_{\alpha}\right) \in \mathbb{C}\left[\mathfrak{a}^{*}\right]^{W_{\circ}}$, the polynomial $\widehat{D}_{\alpha}$ itself need not lie in the image of the mapping $\bar{\rho}$ given in Eqs. 2.1 and 2.3. Thus, we cannot define polynomials $E_{\alpha} \in \mathbb{C}\left[V_{\mathbb{R}}\right]^{K}$ as in Definition 2.1 or obtain the related functions $\varepsilon_{L} \in C\left(\mathfrak{h}_{V}^{*}\right)^{K}$ as claimed in Proposition 4.2.

Lemma A.2 below provides a technical tool needed to revise the proof for Theorem 1.2. First, we require the following substitute for Lemma 2.7 from the paper.

Lemma A.1 For a well-behaved multiplicity free action $K: V$ and $\alpha, \beta \in \Lambda$ one has

$$
\left(\operatorname{top}\left(\widehat{D}_{\alpha}\right)\right)(\beta)=p_{\alpha}\left(v_{\beta}\right) .
$$

Proof Equation 2.3 yields

$$
\bar{\rho}\left(p_{\alpha}\right)(\beta)=\widetilde{p}_{\alpha}(z, \eta(z, \beta))
$$

for any $z \in V$ satisfying $h_{\beta}(z) \neq 0$. Condition (i) in Definition 2.4 allows us to take $z=v_{\beta}$ and condition (ii) gives $\eta\left(v_{\beta}, \beta\right)=\left\langle\cdot, v_{\beta}\right\rangle=v_{\beta}^{*}$. So then

$$
\left(\operatorname{top}\left(\widehat{D}_{\alpha}\right)\right)(\beta)=\bar{\rho}\left(p_{\alpha}\right)(\beta)=\widetilde{p}_{\alpha}\left(v_{\beta}, v_{\beta}^{*}\right)=p_{\alpha}\left(v_{\beta}\right) .
$$

The online version of the original article can be found under doi:10.1007/s10231-011-0234-x.

C. Benson $(\varangle) \cdot$ G. Ratcliff

Department of Mathematics, East Carolina University Greenville,

Greenville, NC 27858, USA

e-mail: bensonf@ecu.edu

G. Ratcliff

e-mail: ratcliffg@ecu.edu 
Lemma A.2 Let $K: V$ be a well-behaved multiplicity free action, $\left(\beta_{n}\right)$ a sequence in $\Lambda$ and $\left(\lambda_{n}\right)$ a sequences in $\mathbb{R}^{\times}$with $\lim \lambda_{n}=0$ and $\left(\left|\lambda_{n}\right| \beta_{n}\right)$ converging in $\mathfrak{a}^{*}$. Then $\lim \widehat{L_{\alpha}}\left(\phi_{\beta_{n}, \lambda_{n}}\right)=\lim p_{\alpha}\left(\sqrt{2\left|\lambda_{n}\right|} v_{\beta_{n}}\right)$ for all $\alpha \in \Lambda$.

Proof Recall that $\widehat{L_{\alpha}}\left(\phi_{\beta_{n}, \lambda_{n}}\right)=\left(2\left|\lambda_{n}\right|\right)^{|\alpha|} \widehat{D}_{\alpha}\left(\beta_{n}\right)$ by Eq. 3.1. So now

$$
\lim \widehat{L_{\alpha}}\left(\phi_{\beta_{n}, \lambda_{n}}\right)=\lim \left(2\left|\lambda_{n}\right|\right)^{|\alpha|} \widehat{D}_{\alpha}\left(\beta_{n}\right)=\lim \left(2\left|\lambda_{n}\right|\right)^{|\alpha|} \widehat{D}_{\alpha}\left(\left(2\left|\lambda_{n}\right|\right)^{-1} \beta\right)=\left(\operatorname{top}\left(\widehat{D}_{\alpha}\right)\right)(\beta)
$$

where $\beta:=\lim \left(2\left|\lambda_{n}\right| \beta_{n}\right)$. On the other hand, using Lemma A.1,

$$
\begin{aligned}
\left.\operatorname{top}\left(\widehat{D}_{\alpha}\right)\right)(\beta) & =\lim \left(\operatorname{top}\left(\widehat{D}_{\alpha}\right)\right)\left(2\left|\lambda_{n}\right| \beta_{n}\right) \\
& =\lim \left(2\left|\lambda_{n}\right|\right)^{|\alpha|}\left(\operatorname{top}\left(\widehat{D}_{\alpha}\right)\right)\left(\beta_{n}\right) \\
& =\lim \left(2\left|\lambda_{n}\right|\right)^{|\alpha|} p_{\alpha}\left(v_{\beta_{n}}\right)=\lim p_{\alpha}\left(\sqrt{2\left|\lambda_{n}\right|} v_{\beta_{n}}\right) .
\end{aligned}
$$

Revised Proof of Theorem 1.2 Assume that the multiplicity free action $K: V$ is well behaved. Let $\left(\varphi_{n}\right)_{n=1}^{\infty}$ be a sequence in $\Delta\left(K, H_{V}\right), \varphi \in \Delta\left(K, H_{V}\right)$, and write $\mathcal{K}_{n}=\Psi\left(\varphi_{n}\right), \mathcal{K}=\Psi(\varphi)$. We will show that

$$
\varphi_{n} \longrightarrow \varphi \text { in } \Delta\left(K, H_{V}\right) \Longleftrightarrow \mathcal{K}_{n} \longrightarrow \mathcal{K} \text { in } \mathfrak{h}_{V}^{*} / K .
$$

The Heisenberg fan model shows that $\varphi_{n} \longrightarrow \varphi$ if and only if $\widehat{L}\left(\varphi_{n}\right) \longrightarrow \widehat{L}(\varphi)$ for every $L \in \mathbb{D}_{K}\left(H_{V}\right)$. By considering subsequences, we may assume that either every $\varphi_{n}$ is a spherical function of Type 2 or every $\varphi_{n}$ is of Type 1 .

$(\Rightarrow)$ : First assume that $\varphi_{n} \longrightarrow \varphi$. We will show $\mathcal{K}_{n} \longrightarrow \mathcal{K}$.

Case 1: Suppose that each $\varphi_{n}$ is a spherical function of Type 2. For some points $w_{n} \in V$, one has $\varphi_{n}=\eta_{K \cdot w_{n}}$ and $\mathcal{K}_{n}=\left(K \cdot w_{n}\right) \times\{0\}$. As $\widehat{T} \varphi=\lim \widehat{T} \varphi_{n}=0$, it follows that $\varphi$ is also of Type 2. So now $\varphi=\eta_{K \cdot w}$ and $\mathcal{K}=(K \cdot w) \times\{0\}$ for some $w \in V$. As $\widehat{L_{\circ}}\left(\varphi_{n}\right)=\left|w_{n}\right|^{2}$ [(see (4.2)] converges to $\widehat{L_{\circ}}(\varphi)=|w|^{2}$, it follows that $\left(w_{n}\right)$ is a bounded sequence. Passing to a subsequence, we may assume that $\left(w_{n}\right)$ converges in $V$, with $\lim w_{n}=w^{\prime}$ say. Now for each $\alpha \in \Lambda$, we observe that

$$
p_{\alpha}\left(w^{\prime}\right)=\lim p_{\alpha}\left(w_{n}\right)=\lim \widehat{L_{\alpha}}\left(\varphi_{n}\right)=\widehat{L_{\alpha}}(\varphi)=p_{\alpha}(w) .
$$

As $\left\{p_{\alpha}: \alpha \in \Lambda\right\}$ is a basis for $\mathbb{C}\left[V_{\mathbb{R}}\right]^{K}$ and the invariants for a compact linear action separate orbits it follows that $K \cdot w^{\prime}=K \cdot w$. Hence, $\mathcal{K}_{n}$ converges to $\mathcal{K}$ in $\mathfrak{h}_{V}^{*} / K$.

Case 2: Suppose that each $\varphi_{n}$ is a spherical function of Type 1,

$$
\varphi_{n}=\phi_{\beta_{n}, \lambda_{n}} \text { and } \mathcal{K}_{n}=\sqrt{2\left|\lambda_{n}\right|}\left(K \cdot v_{\beta_{n}}\right) \times\left\{\lambda_{n}\right\}
$$

say. Let

$$
\lambda:=\widehat{T} \varphi=\lim \widehat{T} \varphi_{n}=\lim \lambda_{n} .
$$

Case 2(a): If $\lambda \neq 0$ then $\varphi=\phi_{\beta, \lambda}$ and $\mathcal{K}=\sqrt{2|\lambda|}\left(K \cdot v_{\beta}\right) \times\{\lambda\}$ for some $\beta \in \Lambda$. As $\widehat{L_{\circ}}\left(\varphi_{n}\right)=2\left|\lambda_{n}\right|\left|\beta_{n}\right|$ converges to $\widehat{L_{\circ}}(\varphi)=2|\lambda||\beta|$, it follows that $\lim \left|\beta_{n}\right|=|\beta|$. As $\{\alpha \in \Lambda:|\alpha|=|\beta|\}$ is a finite set we can assume, by passing to a subsequence, that $\beta_{n}=\beta$ for every $n$. So now $\mathcal{K}_{n}=\sqrt{2\left|\lambda_{n}\right|}\left(K \cdot v_{\beta}\right) \times\left\{\lambda_{n}\right\}$ with $\lambda_{n} \longrightarrow \lambda$ and thus $\mathcal{K}_{n} \longrightarrow \mathcal{K}$ as desired.

Case 2(b): If $\lambda=0$ then $\varphi=\eta_{K} \cdot w$ and $\mathcal{K}=(K \cdot w) \times\{0\}$ for some $w \in V$. Moreover, $\widehat{L_{\circ}}\left(\varphi_{n}\right)=2\left|\lambda_{n}\right|\left|\beta_{n}\right|=2\left|\lambda_{n}\right|\left|v_{\beta_{n}}\right|^{2}$ converges to $\widehat{L_{\circ}}(\varphi)=|w|^{2}$, and thus $\sqrt{2\left|\lambda_{n}\right|} v_{\beta_{n}}$ is a bounded sequence. By passing to a subsequence, we may assume this converges in $V$ and 
write $v:=\lim \sqrt{2\left|\lambda_{n}\right|} v_{\beta_{n}}$. Applying the moment map, it follows that $2\left|\lambda_{n}\right| \beta_{n}$ converges to a point $\beta \in \mathfrak{a}^{*}$ with $\beta_{\mathfrak{k}}=\tau(v)$. Now Lemma A.2 yields

$$
p_{\alpha}(w)=\widehat{L_{\alpha}}(\varphi)=\lim \widehat{L_{\alpha}}\left(\phi_{\beta_{n}, \lambda_{n}}\right)=\lim p_{\alpha}\left(\sqrt{2|\lambda|} v_{\beta_{n}}\right)=p_{\alpha}(v)
$$

for each $\alpha \in \Lambda$. As in Case 1, this implies that $K \cdot w=K \cdot v$ and thus $\mathcal{K}=\lim \mathcal{K}_{n}$.

$(\Leftarrow)$ : Next assume conversely that $\mathcal{K}_{n} \longrightarrow \mathcal{K}$. We will show $\varphi_{n} \longrightarrow \varphi$.

Case 1: Suppose that each $\varphi_{n}$ is a spherical function of Type 2 . Hence $\mathcal{K}_{n} \subset V \times\{0\}$ for all $n$ and as $\mathcal{K}_{n} \rightarrow \mathcal{K}$, it follows that $\mathcal{K} \subset V \times\{0\}$ and that $\varphi$ is of Type 2. So

$$
\varphi_{n}=\eta_{K \cdot w_{n}}, \mathcal{K}_{n}=\left(K \cdot w_{n}\right) \times\{0\} ; \quad \varphi=\eta_{K \cdot w}, \mathcal{K}=(K \cdot w) \times\{0\}
$$

say. Now $K \cdot w_{n} \longrightarrow K \cdot w$ and hence $\left(\widehat{L_{\alpha}}\left(\varphi_{n}\right)=p_{\alpha}\left(w_{n}\right)\right) \longrightarrow\left(p_{\alpha}(w)=\widehat{L_{\alpha}}(\varphi)\right)$ for each $\alpha \in \Lambda$. It follows that $\varphi_{n} \longrightarrow \varphi$.

Case 2: Suppose that each $\varphi_{n}$ is a spherical function of Type 1, and write

$$
\varphi_{n}=\phi_{\beta_{n}, \lambda_{n}} \text { and } \mathcal{K}_{n}=\sqrt{2\left|\lambda_{n}\right|}\left(K \cdot v_{\beta_{n}}\right) \times\left\{\lambda_{n}\right\} .
$$

As $\left(\mathcal{K}_{n}\right)$ converges so does $\left(\lambda_{n}\right)$. Let $\lambda:=\lim \lambda_{n}$.

Case 2(a): If $\lambda \neq 0$ then $\varphi=\phi_{\beta, \lambda}$ and $\mathcal{K}=\sqrt{2|\lambda|}\left(K \cdot v_{\beta}\right) \times\{\lambda\}$ for some $\beta \in \Lambda$ with $K \cdot v_{\beta_{n}} \longrightarrow K \cdot v_{\beta}$. As $\Lambda$ is a discrete set it follows that the sequence $\left(\beta_{n}\right)$ is eventually constant. Thus $\varphi=\phi_{\beta, \lambda}=\lim \phi_{\beta, \lambda_{n}}=\lim \varphi_{n}$.

Case 2(b): If $\lambda=0$ then $\varphi=\eta_{K} \cdot w$ and $\mathcal{K}=(K \cdot w) \times\{0\}$ for some $w \in V$. As $\mathcal{K}_{n} \longrightarrow \mathcal{K}$, we have $\sqrt{2\left|\lambda_{n}\right|}\left(K \cdot v_{\beta_{n}}\right) \longrightarrow K \cdot w$ and by passing to a subsequence we may assume that $\sqrt{2\left|\lambda_{n}\right|} v_{\beta_{n}}$ converges to a point $v \in K \cdot w$. Applying the moment map, it follows that $2\left|\lambda_{n}\right| \beta_{n}$ converges in $\mathfrak{a}^{*}$. Again using Lemma A.2, we obtain

$$
\lim \widehat{L_{\alpha}}\left(\varphi_{n}\right)=\lim p_{\alpha}\left(\sqrt{2\left|\lambda_{n}\right|} v_{\beta_{n}}\right)=p_{\alpha}(v)=p_{\alpha}(w)=\widehat{L_{\alpha}}(\varphi)
$$

for each $\alpha \in \Lambda$ and hence $\varphi_{n} \longrightarrow \varphi$ as claimed.

Finally, we note that without the functions $\varepsilon_{L}$ given by Proposition 4.2 we do not obtain an explicit homeomorphism between the orbital and Heisenberg fan models for $\Delta\left(K, H_{V}\right)$, as claimed in Corollary 4.4 . 